\title{
Scientific Basis for Regulatory Decision- Making of Nanomaterials Report on the Workshop, 20-21 January 2014, Center of Applied Ecotoxicology, Dübendorf
}

\author{
Christoph Studer ${ }^{\mathrm{a}}$, Lothar Aicher ${ }^{\mathrm{b}}$, Bojan Gasic ${ }^{\mathrm{c}}$, Natalie von Goetz ${ }^{\mathrm{d}}$, Peter Hoet ${ }^{\mathrm{e}}$, \\ Jörg Huwylerf, Ralf Kägi', Robert Kase ${ }^{\text {h }}$, Andrej Kobe', Bernd Nowackj, \\ Barbara Rothen-Rutishauserk, Kristin Schirmer', Gregor Schneiderm, Etienne Vermeissen ${ }^{\mathrm{h}}$, \\ Peter Wicki, and Tobias Walser*a
}

\begin{abstract}
The key findings of a workshop jointly organized by the Swiss Centre of Applied Ecotoxicity, the Swiss Centre for Applied Human Toxicology (SCAHT), and the Federal Office of Public Health (FOPH) are summarized and provide a critical analysis of the current regulatory framework for nanomaterials and a snapshot of some hot topics in nanoscience.
\end{abstract}

Keywords: Nanomaterials · Regulation of nanomaterials $\cdot$ Risk assessment

\section{Introduction}

Nanomaterials are an important aspect of nanotechnology and benefit our daily lives in many ways. Surfaces, sizes and structures on the nanoscale can be tuned virtually infinitely, which allows for innovative applications e.g. in medicine and engineering. For instance, material and energy intensity is lowered by adding nanomaterials to plastics. The use of nanomaterials in pharmaceuticals allows specifically targeted, cutting edge medical treatment. A primary consequence is that the amount of active compounds can be

\footnotetext{
${ }^{*}$ Correspondence: Dr. T. Walsera,

Tel.: +415846386 64,

E-mail: tobias.walser@bag.admin.ch

a Federal Office of Public Health FOPH, Bern,

Switzerland; ' ${ }^{\text {S }}$ wiss Centre for Applied Human

Toxicology, University of Basel, Switzerland;

' State Secretariat for Economic Affairs Seco,

Bern, Switzerland; ' Swiss Federal Institute of

Technology ETHZ, Zurich, Switzerland; e Univer-

sity of Leuven, Leuven, Netherlands; ' University

of Basel, Basel, Switzerland; " Swiss Federal

Laboratories for Materials Science and Techno-

logy, Dübendorf, Switzerland; ${ }^{h}$ Ecotox Centre,

Dübendorf, Switzerland; 'European Commission,

Brussels, Belgium; 'Swiss Federal Laboratories

for Materials Science and Technology, St.Gallen,

Switzerland; ' Adolphe Merkle Institute, Fribourg,

Switzerland; 'Swiss Federal Institute of Aquatic

Science and Technology, Dübendorf, Switzerland;

mRAS Materials, Regensburg, Germany
}

reduced, thus minimizing side-effects. On the other hand, the use of nanomaterials can lead to risks, which are not encountered with bulk counterparts. While intact skin is impermeable for nanomaterials, some of them can, if inhaled, reach the alveoli in the lungs and translocate into the blood stream with subsequent accumulation in the organs. Such exposure can give rise to adverse local and systemic health effects. Beside potential human toxicity, ecotoxic effects caused by certain nanomaterials have been detected in laboratory and field studies. The properties of nanomaterials are often different compared to those of their bulk counterparts, which poses a challenge for researchers, but also regulators. Regulatory frameworks for conventional chemicals may, therefore, not be entirely valid for nano-specific properties and consequently need to be adapted.

In order to refresh and update the knowledge of regulators and researchers, the Swiss Centre of Applied Ecotoxicity, the Swiss Centre for Applied Human Toxicology (SCAHT), and the Federal Office of Public Health (FOPH) jointly organized a public workshop. 50 participants followed the presentations and workshops on exposure science, human- and ecotoxicology, and case studies from industry. The use of the latest findings from these fields for risk assessment was illustrated with examples and subsequently discussed in break-out groups. This article summarizes the key findings of the workshop and provides a critical analysis of the current regulatory framework for nanomaterials.

\section{Regulation of Nanomaterials}

\subsection{Nanospecific Regulation}

Nanomaterials are regulated under different regulatory regimes, depending on their field of application. The primary goal is to ensure the safety of the nano-enabled applications for workers, consumers and the environment. The current nano-specific requirements in the European Union (EU) and Switzerland $(\mathrm{CH})$ are summarized in Table 1.

An important regulatory constituent is the definition of the term 'nanomaterial'. It provides the key for subsequent nanospecific regulatory requirements. Most of the current definitions use a consensus value of $100 \mathrm{~nm}$ (see box 1). In addition to size, specific surface area and shape are used to define nanomaterials. Sometimes further classifiers are applied, for example, minimal percentages of nanoscale particles; synthetic nature; or that it must have a nano-specific function. The various definitions are under constant discussion because of the different stakeholder perspectives. From a regulatory perspective, an upper size limit has the advantage of being easily controllable and hence enforceable; in contrast to definitions that include a nano-specific function or the prerequisite that nanomaterials must be man-made.

Ideally, the term 'nanomaterial' should embrace all materials with nano-specific properties that need to be considered in a regulatory risk assessment. However, not all nano-specific properties are exhaustively known yet. A solution might be a 
precautionary, broad definition, combined with a corresponding evaluation and test strategy. This would reduce the test efforts, which are currently conducted on a caseby-case basis.

In EU, the EU definition of nanomaterials, adopted in 2011, has gathered considered traction. Experience is currently being reviewed. Furthermore, there is ongoing discussion on nano-specific amendments to the 'Novel Food Regulation' and the annexes of the Regulation on Registration, Evaluation, Authorization and Restriction of Chemicals REACH.[1] In Switzerland, harmonization of the Foodstuffs Act with European foodstuff regulations is ongoing. This includes the discussion on labeling for nanomaterials in cosmetics and food. Further information on differences and commonalities between Swiss and European legislation are shown in Table 1.

\subsection{Regulatory Tools to Support the Safety of Nanomaterials}

A variety of tools are available to regulators in order to support the safety of people and the environment. Chemicals that lead to exposure situations with adverse outcomes are prohibited or their use is restricted. Further regulatory measures for biologically active agents in pharmaceuticals, biocides and plant protection products, as well as for food supplements and cosmetics, include authorization procedures and lists of approved/forbidden substances. Prior to the introduction of industrial chemicals onto the market, the authority receives information on hazards and risks associated with the substance. The data requirements for the registration dossiers depend on the market volume of the new substance and are the same in the EU and Switzerland. The burden of proof for the safety of the chemical rests with the producer or importer, but the authority checks the risk evaluations. The principle of producers' responsibility applies to substances, as well as to mixtures.

An important safety measure is the information provided to consumers and industry about potential hazards and precautionary measures to be taken. The tools used include classification schemes, labeling, and information sent along the supply chain with safety data sheets. These instruments are also used for nanomaterials. A review is currently under way to check whether classification schemes for chemicals are applicable also for nanomaterials. ${ }^{[2]}$

\subsection{Prerequisites for Nano- specific Amendments of Existing Regulations}

Nanomaterials are regulated already today (see Table 1). However, there are gaps regarding nano-specific data requirements in the existing registration and authorization schemes. Scientific evidence forms the basis of data on any hazards that registrants are required to supply. This data, together with information on exposure, is used to conduct the human and environmental risk assessment. Today it has to be decided on a case-by-case basis what additional data are needed for nanomaterials. Prior to standardizing the exact nano-specific requirements for the dossiers, further knowledge and consensus is required:

Identity: According to the present Swiss chemicals legislation, nanomaterials are not only nanomaterials, but they also fall under the definition of a substance. Elemental composition defines a substance in the chemicals legislation. The definition does not take into account size, shape, and the potentially complex constitution of a multi-component nanomaterial. Hence, it is difficult to distinguish between different nanomaterials and classify them as either an individual substance, forms of bulk substances or as a mixture. Sound rules on the identity of nanomaterials would clarify when a registration, including testing, is necessary. Furthermore, a grouping of nanomaterials according to e.g. their physico-chemical properties or toxicological characteristics would reduce testing efforts (an example is given in Fig. 1).

Characterization: To date a harmonized set of required physical-chemical

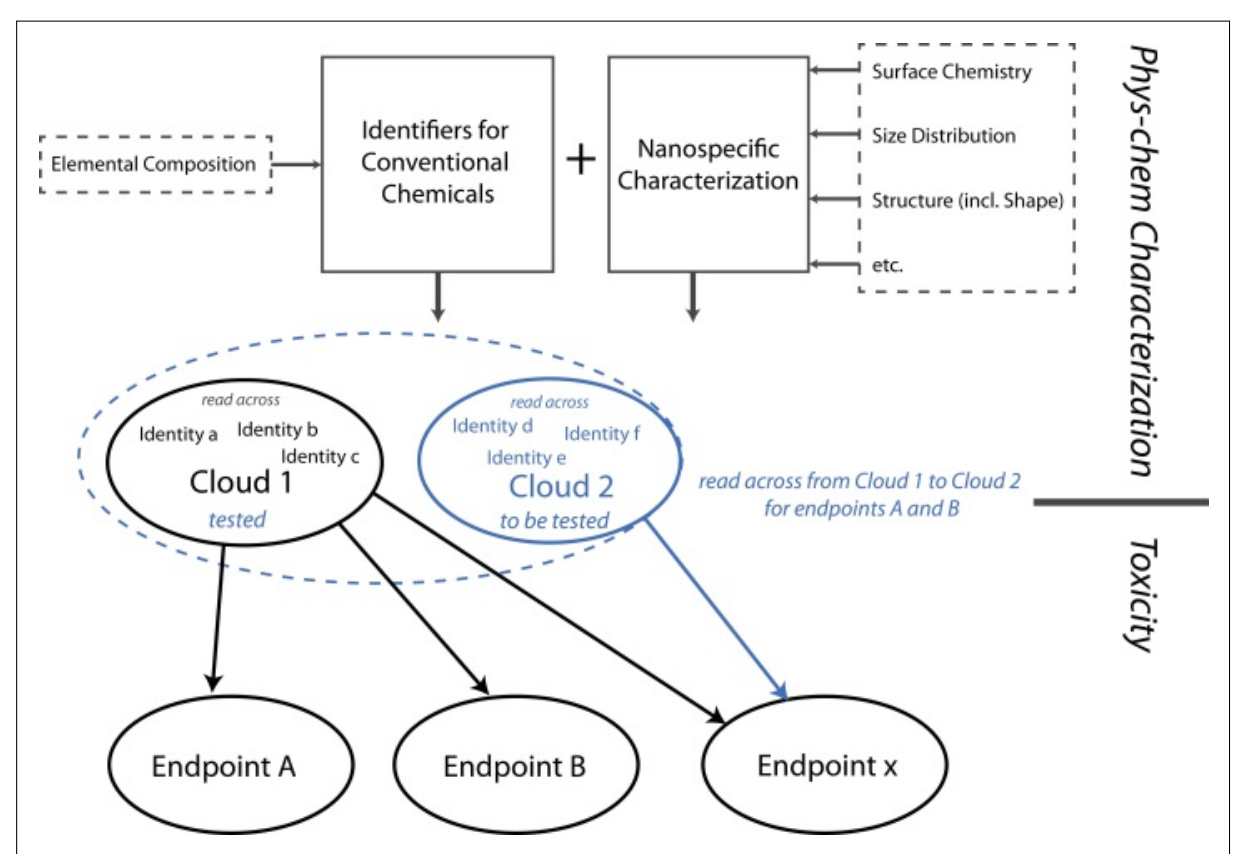

Fig. 1. Nanomaterials require additional characterization in comparison to conventional chemicals. After their characterization, they can be associated with predefined clouds of similar nanomaterials that require the same testing strategy. Some clouds behave similarly for a particular endpoint, which allows to read across clouds for this specific end point (dotted oval, in this example endpoint $A$ and $B$ for Cloud 1 and 2). Therefore, testing efforts can be significantly decreased the more information is known. In the example above, Cloud 2 needs only to be tested on Endpoint(s) $x$, because information from Cloud 1 can be used for endpoint $A$ and $B$ (but not for endpoint $\mathrm{x}$ ). A prerequisite for an efficient testing strategy is a validated grouping scheme. 


\section{EU}

Food additives $^{\mathrm{a}}$

Materials and articles made from plastic which come into contact with foodstuffs ${ }^{\mathrm{b}}$

Biocidal products ${ }^{\mathrm{c}}$

Cosmetics $^{\mathrm{e}}$

Chemicals $^{\mathrm{f}}$

Information on food packaging for consumers $^{\mathrm{g}}$

\section{Switzerland}

Chemicals

Plant protection products $^{\mathrm{j}}$

Biocidal products ${ }^{\mathrm{k}}$

Pharmaceuticals

\section{Authorization}

Nanoscale additives and ingredients require an evaluation and authorization.

Nanoscale active compounds require an evaluation and authorization

Nano cosmetics: specification of the nanoscale ingredient and safety information

Registration of nanomaterial as new substance or form of the bulk substance

\section{Registration}

Two separate cases according to $\mathrm{ChemO}^{\mathrm{h}}$ : Registration for new substances and obligation to notify old substances

Registration: The dossier contains a comprehensive characterization of nanomaterials.

Obligation to notify and comprehensive characterization of nanomaterials if present as hazardous old substances and in mixtures
Information labeling (Nano) and hazard classification and labeling according to CLP $^{\mathrm{d}}$

Infolabel (Nano)

Hazard classification and labeling according to CLP ${ }^{d}$

Information labeling (Nano)

\section{Labeling}

Hazard classification and labeling according to $\mathrm{ChemO}^{\mathrm{i}}$
The dossier contains characterization of the nanomaterials

Nanoscale active compounds require an evaluation and authorization

Application for authorization or revision must state whether the product contains nanomaterials
Hazard classification and labeling according to $\mathrm{ChemO}^{\mathrm{i}}$

Hazard classification and labeling according to $\mathrm{ChemO}^{\mathrm{i}}$

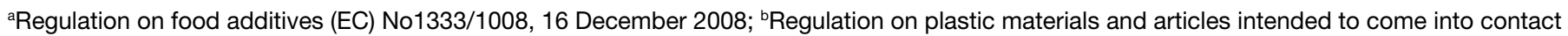

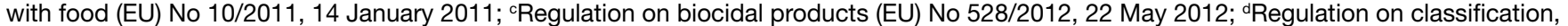
labelling and packaging of substances and mixtures (EC) No 1272/2008, 16 December 2008; eRegulation on cosmetic products (EC) No 1223/2009, 30 November 2009; ${ }^{R}$ EACH does not have any nano-specific requirements yet. However, the European commission published a guidance document on how nanomaterials should be treated under REACH: Nanomaterials in REACH, CA/59/2008 rev.1, Brussels, 16 December 2008; ${ }^{9}$ Regulation on the provision of food information to consumers (EU) No 1169/2011, 25 October 2011); ${ }^{\circ}$ Chemicals Ordinance Art. 16 and Art. 61 (SR 813.11); ' Chemicals Ordinance Art. 8 and Art. 34b (SR 813.11); i Ordinance on Plant Protection Products (SR 916.161); ${ }^{\circ}$ Ordinance on Biocidal Products (SR 813.12).

standard media, the applied dose and cell types. Such reproducible methods can then become part of an integrated test strategy, which will ultimately serve as a supporting tool for regulatory decisions on the safety of nanomaterials. In this context it is important to elucidate modes of actions responsible for long-term effects, and to develop in vitro systems to detect them. Read-across and QSARs (Quantitative Structure-Activity Relationships) are part of such a test strategy as they can make use of the same toxicity data for similar nanomaterials and be used to predict the toxicity of nanomaterials from a limited set of physico-chemical data.

Exposure models and bioaccumulation: Existing exposure models for con- 
ventional chemicals are of limited value for nanomaterials. For instance, the often used partition coefficients $\log \mathrm{P}_{\text {ow }}$ or $\log$ $\mathrm{K}_{\mathrm{oc}}$ cannot be used for nanomaterials. ${ }^{[3]}$ In contrast, homo-agglomeration, heteroagglomeration and sedimentation are parameters which are very relevant for nanomaterials, and their inclusion would mark an important step towards better nanospecific fate and exposure models. In addition, the estimation of bioaccumulation and biomagnification of nanomaterials in the food chain is still conducted on a case-by-case basis, and the respective modeling is at an early stage. Pharmacokinetic models for conventional chemicals are a good starting point to estimate the burden of nanomaterials in organs and organisms, but they need to be adapted to nanomaterials. Once steady-state nanomaterial concentrations in the organs are estimated, they can be compared with toxicity data from in vitro tests for the same nanomaterials in order to assess the risk.

\section{Short Summaries of the Presentations from Science and Industry}

\subsection{Environmental Exposure}

A variety of emission sources release nanomaterials via wastewater, air or other pathways into the environment. Measurements and fate models can measure or estimate the resulting nanoparticle concentrations in the environment. Today, only few data exist on quantitative release and nanomaterial ageing. Ageing refers to the typical phenomena whereby pristine nanomaterials undergo physical and chemical changes during their lifetime, affecting among other things their toxicity over time, therefore complicating risk assessment. Nanoparticle concentrations in the environment have been probabilistically modeled for silver, titanium dioxide, zinc oxide, carbon nanotubes (CNTs) and fullerenes in order to account for different applications and processes along the lifecycle of engineered nanomaterials. ${ }^{[4]}$ Compared with effect concentrations from laboratory studies, emitted nanosilver, which ends up in rivers, may reach harmful concentrations in worst case scenarios (high input, low dilution). ${ }^{[5]}$ Unfortunately, such modeling results are difficult to validate because current analytical capabilities for the distinction between natural and man-made nanomaterials in complex media and very low concentrations like in river water are very costly and labor intensive. For instance, enrichment and separation of the larger fraction is a daunting prerequisite for such an analysis. A complicating factor is agglomeration and - again - ageing of the nanomaterials. ${ }^{[6]}$

\subsection{Human Exposure}

Free nanomaterials can be taken up via different uptake routes. Potential sources are silver nanomaterials in textiles, food containers (forbidden in the EU), or pharmaceuticals (sprays, tablets). Released nanomaterials can subsequently migrate to food or skin with potential uptake into the human body. A pharmacokinetic model allows the estimation of nanomaterial concentrations in organs that result from defined exposure. The calculated concentrations can then be compared to toxicity data. An example of a pharmacokinetic model was demonstrated with nanosilver as reference material. ${ }^{\text {[7] }}$ Different steady-state concentrations for the organs were modeled, influenced by uptake route and exposure situations. The comparison with in vitro toxicity data showed that realistic exposure situations with products on the market do not result in an alerting consumer exposure, but that exposure to nanomaterial producers in workplaces may need particular attention.

\subsection{Ecotoxicology and Bioaccumulation}

Different nanomaterials lead to different effects in organisms. The acute toxicity of metallic nanomaterials seems to be similar to the corresponding metal ions or bulk counterparts. The same holds true for organic nanomaterials which exhibit toxicities of the same magnitude as the corresponding conventional chemicals ${ }^{[8]}$ though particle toxicity in addition to ion toxicity has however been identified also in metallic nanoparticles. ${ }^{\text {[9] }}$ Such particle specific effects are becoming increasingly known. For instance, titanium dioxide $\left(\mathrm{TiO}_{2}\right)$ nanomaterials can influence the functioning of microbial digestion enzymes in biofilms ${ }^{[10]}$ and nano-cerium dioxide $\left(\mathrm{CeO}_{2}\right)$ influences the growth of rhizobium in soy. ${ }^{[11]}$ Compared to toxicity, data on bioaccumulation is scarcer. Results indicate that for silver, the bioaccumulation potential is the same for both the nano and ionic form. However, the actual type of test system applied is of key importance in evaluating bioaccumulation potential. Daphnia, for instance, can accumulate nanomaterials in their gut even though they are not taken up by the gut cells into the organism. Hence, it is not a true bioaccumulation.

\subsection{Human Toxicology}

Whether nanomaterials exhibit new modes of toxic action in comparison to bulk materials is a controversially discussed question. A study aimed at (partly) answering this question used silica of different sizes (100-200 nm) and different surface functionalizations in order to determine potential effects on human cells.
End points were cell viability, induction of reactive oxygen species (ROS) in hepatocytes and macrophages, and hemolysis. Results show a concentration dependent effect and a correlation between cell uptake and toxicity. ${ }^{[12]}$ The strongest effect on cell viability and hemolysis was induced by negatively charged, mesoporous, relatively large particles, which disintegrated upon cell uptake. Hemolysis indicates a direct effect of the nanomaterials on the plasma membrane of red blood cells. This effect is currently being further investigated. No ROS was detected. Despite the robustness of the study results, it is not yet possible to interpret a potential impact on human health.[13]

\subsection{Screening Methods}

Robust and meaningful test methods are a prerequisite in evaluating nanomaterial toxicity. ${ }^{[14]}$ In vitro methods are an essential part of a test strategy to improve toxicity assessment and to set testing priorities for certain classes of nanomaterials. Therefore, such methods are currently being developed and pre-validated in order to cover relevant end points for nanomaterials. ${ }^{[15]}$ Research foci are laid on cell or small-scale organism viability assays, detection of ROS induction, ${ }^{[16]}$ pro-inflammatory markers and genotoxicity assays..$^{[17]}$ In order to mimic the lung, which is an important portal of entry for nanomaterials, a 3D-cell culture model has been developed.[18] The cell model was used to estimate the toxicity of silver nanomaterials from typical occupational exposure situations. [19] No effects on cell morphology, cytotoxicity, ROS or inflammation were detected after single exposure. Adverse effects only occurred at nanosilver concentrations much higher than typically expected at workplaces.

\subsection{Case Study Nanosilver}

The EU Scientific Committee on Emerging and Newly Identified Health Risks (SCENIHR) published the opinion 'Nanosilver: Safety and environmental effects and role in antimicrobial resistance' in December 2013. ${ }^{[20]}$ The aim of this opinion was to assess whether the use of nanosilver, in particular in medical care and in consumer products, could result in additional risks compared to more traditional uses of silver and whether the use of nanosilver to control bacterial growth could result in resistance of micro-organisms. SCENIHR concludes that the widespread (and increasing) use of silver-containing products implicates that both consumers and the environment are exposed to new sources of silver. Human exposure is direct (food, hand-to-mouth contact, skin) and may be life-long; while in the environment silver nanomaterials may be a particularly effec- 
tive delivery system for silver to organisms in soil, water and sediment and may act as sources of ionic silver over extended periods of time. Therefore, additional effects caused by widespread and long term use of silver nanomaterials cannot be ruled out. Regarding the hazard associated with the dissemination of the resistance mechanism following the use of silver nanomaterials, more data are needed to better understand bacterial response to ionic silver and silver nanomaterials exposure.

Nanosilver is widely used as an antimicrobial substance. The benefits and risks of nanosilver applications have been demonstrated at this workshop by a company with the example of nanosilver applied to textiles. Reduced amounts of silver and a high washing resistance of nanosilver in textiles as compared to attached micronized droplets is resulting in less material requirements for the same antimicrobial efficacy. Virtually no silver particles were emitted from tested plastics and microfiber textiles. Ecotoxicity and other toxicity tests showed that it is possible to handle nanosilver applications safely.

\section{Conclusion}

Nanomaterials show a variety of properties and have many applications. Therefore, they fall under different regulatory statutes. Testing obligations are principally the same as for conventional chemicals nowadays. However, the tests need to be adapted and new tests need to be developed to take into account nano-specific properties. Unfortunately, standardization and harmonization of the test systems is yet to be implemented. Consequently, case-by-case risk assessment of nanomaterials is still the only option. This creates uncertainty for industry and the authorities and, in addition to the potentially substantial testing efforts, could result in barriers to innovation.

These challenges are recognized by the authorities. They are therefore striving to find efficient solutions. For instance the discussion on the nano-specific requirements in the REACH annexes is ongoing. In parallel, the OECD is working on an update of test guidelines and guidance documents in order to facilitate the implementation of new nano-specific test requirements. ${ }^{[21]}$ The workshop underlined the urgent need to continue research on the exposure to and hazard of nanomaterials in order to provide the basis for a standardized risk assessment of nanomaterials. The ongoing National Research Programme 64 on 'Opportunities and Risks of Nanomaterials', as well as European research programs, will provide some of the knowledge that is lacking. A continuation

\section{Box 1}

The European Union released a proposal for a definition of the term 'nanomaterial' in 2011. This definition is used in many regulations of the EU. Switzerland has in the legislation for chemicals a separate definition which is similar to the EU definition. For the Swiss Medical Legislation, the recommended definition from the US Food and Drug Administration (FDA) is adopted.

- EU Definition (Summary, Recommendation L 275/38, 2011): 'Nanomaterial' means a natural, incidental or manufactured material containing particles, in an unbound state or as an aggregate or as an agglomerate and where, for $50 \%$ or more of the particles in the number size distribution, one or more external dimensions is in the size range $1-100 \mathrm{~nm}$. In specific cases and where warranted by concerns for the environment, health, safety or competitiveness the number size distribution threshold of $50 \%$ may be replaced by a threshold between 1 and $50 \%$. Fullerenes, graphene flakes and single wall carbon nanotubes with one or more external dimensions below $1 \mathrm{~nm}$ should be considered as nanomaterials. A material should be considered as falling under the definition where the specific surface area by volume of the material is greater than $60 \mathrm{~m}^{2} / \mathrm{cm}^{3}$. However, a material which, based on its number size distribution, is a nanomaterial should be considered as complying with the definition even if the material has a specific surface area lower than $60 \mathrm{~m}^{2} / \mathrm{cm}^{3}$.

- Definition according to the Swiss Chemicals Ordinance (SR 813.11): Nanomaterial: Nanomaterial means a material containing particles in an unbound state or as an aggregate or as an agglomerate, where one or more external dimensions is in the size range $1-100 \mathrm{~nm}$, or a material where the specific surface area by volume is greater than $60 \mathrm{~m}^{2} / \mathrm{cm}^{3}$. A material is only considered to be a nanomaterial if it is deliberately produced to utilize the properties arising from the defined external dimensions of the particles it contains, or from the defined surface area by volume of the material. Fullerenes, graphene flakes and single wall carbon nanotubes with one or more external dimensions below $1 \mathrm{~nm}$ are considered to be nanomaterials.

- For the authorization and for adaptations of already registered pharmaceuticals, the applicant must state whether nanomaterials are present in the product. The definition is as follows:

Nanomaterial: At least one dimension 1-1000 nm, and a nanospecific function or mode of action.

of the dialogue between industry, research, the public and the authorities is important in this quickly changing field. It will support the agreement of working priorities, the creation of new insights which will be incorporated in the jurisdictions, and which will be essential for the safe use of nanomaterials.

\section{Received: November 27, 2014}

[1] European Commission, 'Second Regulatory Review on Nanomaterials (572, final)', 2012.

[2] UNECE GHS Sub Committee, 'Review of the applicability of GHS to nanomaterials', ST/SG/ AC.10/C.4/2014/9, 2014.

[3] European Chemicals Agency (ECHA), 'Guidance on information requirements and chemical assessment', Chapter R.16: 'Environmental Exposure Estimation Version 2.1', 2002.

[4] T. Y. Sun, F. Gottschalk, K. Hungerbühler, B. Nowack, Environ. Pollut. 2014, 185, 69.

[5] F. Gottschalk, T. Y. Sun, B. Nowack, Environ. Pollut. 2013, 181, 287.

[6] B. Thalmann, A. Voegelin, B. Sinnet, E. Morgenroth, R. Kaegi, Environ. Sci. Technol. 2014, 48,4885 .

[7] G. Bachler, N. von Goetz, K. Hungerbuehler, Int. J. Nanomed. 2013, 8, 3365 .

[8] A. Kahru, H.-C. Dubourguier, Toxicology 2010, $269,105$.

[9] W. S. Cho, R. Duffin, C. A. Poland, A. Duschl, G. A. Oostingh, W. Magnee, M. Bradley, I. L. Megson, K. Donaldson, Nanotoxicology 2012, $6,22$.
[10] H. Schug, C. W. Isaacson, L. Sigg, A. A. Ammann, K. Schirmer, Environ. Sci. Technol. 2014, 48,11620

[11] J. H. Priester, Y. Ge, R. E. Mielke, A. M. Horst, S. C. Moritz, K. Espinosa, J. Gelb, S. L. Walker, R. M. Nisbet, Y. J. An, Proc. Nat. Sci. 2012, 109, E2451.

[12] S. Seeland, M. Türük, H. Kettiger, A. Treiber, M. Hafner, J. Huwyler, Toxicol. In Vitro 2013, 27, 1109.

[13] H. Kettiger, A. Schipanski, P. Wick, J. Huwyler, Int. J. Nanomed. 2013, 8, 3255.

[14] A. G. Oomen, P. M. J. Bos, T. F. Fernandes, K. Hund-Rinke, D. Boraschi, H. J. Byrne, K. Aschberger, S. Gottardo, F. von der Kammer, D. Kühnel, D. Hristozov, A. Marcomini, L. Migliore, J. Scott-Fordsmand, P. Wick, R. Landsiedel, Nanotoxicology 2014, 8, 334.

[15] R. Schins, A. Knaapen, Inhalation Toxicol. 2007, 19, 189.

[16] M. Roesslein, C. Hirsch, J. P. Kaiser, H. F. Krug, P. Wick, Int. J. Mol. Sci. 2013, 14, 24320.

[17] C. Hirsch, J. P. Kaiser, F. Wessling, K. Fischer, M. Roesslein, P. Wick, H. F. Krug, J. Physics: Conference Series 2011, p. 012053.

[18] B. M. Rothen-Rutishauser, S. G. Kiama, P. Gehr, Am. J. Resp. Cell. Mol. Biol. 2005, 32, 281.

[19] F. Herzog, M. J. Clift, F. Piccapietra, R. Behra, O. Schmid, A. Petri-Fink, B. M. RothenRutishauser, Part. Fibre Toxicol. 2013, 10, 11.

[20] EU Scientific Committee on Emerging and Newly Identified Risks (SCENIHR), 2013.

[21] OECD, 'Recommendation of the Council on the Safety Testing and Assessment of Manufactured Nanomaterials C 107', 2013. 